\title{
Pressure and water quality integrated sensor placement considering leakage and contamination intrusion within water distribution \\ systems
}

Tianwei Mu'a Manhong Huanga,b*, Haoqiang Tanº, Gang Chenª, Rui Zhang ${ }^{\mathrm{d}}$

${ }^{a}$ College of Environmental Science and Engineering, State Environmental Protection Engineering Center for Pollution Treatment and Control in Textile Industry, Donghua University, Shanghai 201620, China;

${ }^{b}$ Shanghai Institute of Pollution Control and Ecological Security, Shanghai 200092, China;

'School of Civil Engineering \& Architecture, Taizhou University, Taizhou, Zhejiang, 318000, China;

${ }^{d}$ School of Hydraulic Engineering, Dalian University of Technology, Dalian, 116024, China;

*College of Environmental Science and Engineering, State Environmental Protection Engineering Center for Pollution Treatment and Control in Textile Industry, Donghua University, Shanghai 201620, China; E-mail:huangmanhong@dhu.edu.cn 


\section{CONTENTS}

Supplementary methods

S1. Trial-and-error principle

S2. Comparison of the clustering model

S3. A case of the backup archive

S4. Example of non-dominated sorting

S5. K-means model

S6. GMM model

S7. Details of comparison

S8. The original data

S9. The system environment

Supplementary Tables (S1-S4)

Supplementary Figures (S1-S6)

Supplementary references 


\section{Tables}

Table S1 The Junction classes via FCM clustering for the EXA7 network.

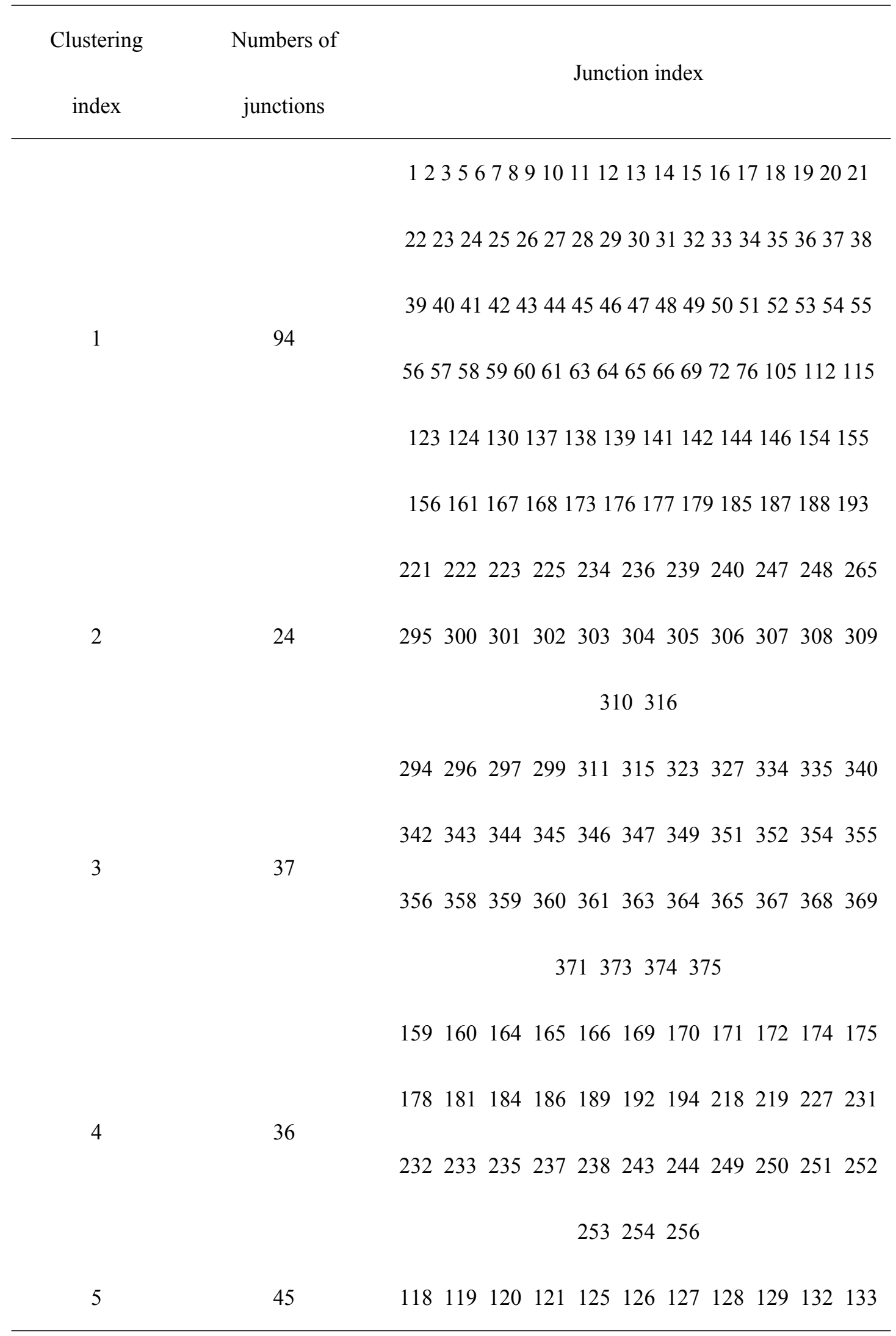


$\begin{array}{lllllllllll}134 & 135 & 136 & 140 & 143 & 145 & 147 & 148 & 149 & 150 & 151\end{array}$

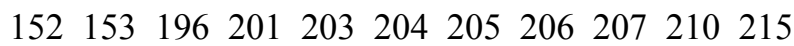

$216217220224226228229230241242 \quad 245$

246

258259260261267268272274276277278

$\begin{array}{lllllllllll}279 & 283 & 284 & 288 & 291 & 312 & 313 & 314 & 317 & 318 & 319\end{array}$

6

8

9

10
38

$\begin{array}{lllllllllll}320 & 321 & 322 & 324 & 325 & 326 & 328 & 329 & 330 & 331 & 332\end{array}$

333336337339348

$\begin{array}{lllllllllll}338 & 341 & 350 & 353 & 357 & 362 & 366 & 370 & 372 & 376 & 377\end{array}$

15

$\begin{array}{llll}378 & 379 & 380 & 381\end{array}$

209212213214255257262263264266269

$\begin{array}{lllllllllll}270 & 271 & 273 & 275 & 280 & 281 & 282 & 285 & 286 & 287 & 289\end{array}$

290292293298

$\begin{array}{lllllllllll}79 & 81 & 82 & 83 & 84 & 88 & 91 & 93 & 94 & 98 & 99\end{array}$

$\begin{array}{lllllllllll}100 & 104 & 106 & 107 & 111 & 113 & 114 & 157 & 158 & 162 & 163\end{array}$

35

$\begin{array}{lllllllllll}180 & 182 & 183 & 190 & 191 & 195 & 197 & 198 & 199 & 200 & 202\end{array}$

208211

$\begin{array}{lllllllllll}4 & 62 & 67 & 68 & 70 & 71 & 73 & 74 & 75 & 77 & 78\end{array}$

$\begin{array}{lllllllllll}80 & 85 & 86 & 87 & 89 & 90 & 92 & 95 & 96 & 97 & 101\end{array}$

$\begin{array}{lllllllll}102 & 103 & 108 & 109 & 110 & 116 & 117 & 122 & 131\end{array}$ 
Table S2 Sensor placement solution in various failure scenarios for the EXA7 network.

\begin{tabular}{ccccccccccc}
\hline Scenario & $c 1$ & $c 2$ & $c 3$ & $c 4$ & $c 5$ & $c 6$ & $c 7$ & $c 8$ & $c 9$ & $c 10$ \\
\hline 0 & 32 & 301 & 354 & 251 & 245 & 322 & 370 & 275 & 94 & 4 \\
1 & - & 304 & 323 & 238 & 220 & 261 & 341 & 275 & 107 & 4 \\
2 & 46 & 240 & - & - & 216 & 268 & 341 & 275 & 211 & 70 \\
3 & - & - & 346 & 218 & - & 261 & 370 & 214 & 182 & 4 \\
4 & - & - & - & 235 & 205 & 283 & - & 282 & 182 & 4 \\
5 & - & - & 334 & 218 & - & - & 362 & 285 & 113 & - \\
6 & 46 & - & 323 & 218 & - & - & - & 290 & - & - \\
7 & 40 & 308 & 344 & - & - & - & - & - & - & - \\
8 & 46 & - & - & - & - & 261 & - & - & - & - \\
9 & - & - & - & - & 216 & - & - & - & - & - \\
\hline
\end{tabular}

c1 represents class 1 of FCM clustering.

The column contents of $\mathrm{c} 1$ to $\mathrm{c} 10$ are the node index.

The scenario represents a sensor failure scenario.

The column contents of the scenario are the number of failure nodes.

"-"represents this sensor is a failure.

Table S3 The junction classes via FCM clustering for the HaiN town.

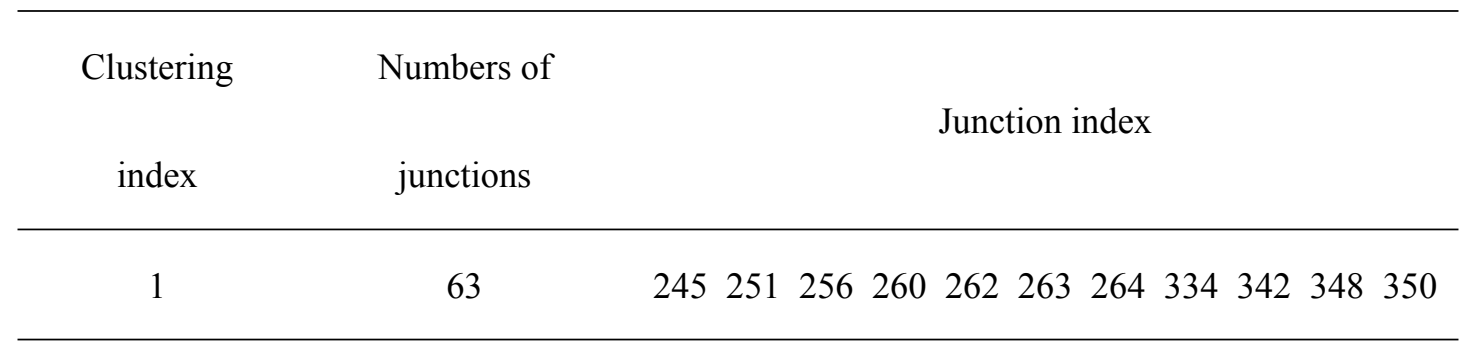




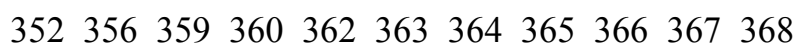

$\begin{array}{lllllllllll}369 & 370 & 371 & 372 & 373 & 374 & 375 & 376 & 377 & 378 & 379\end{array}$

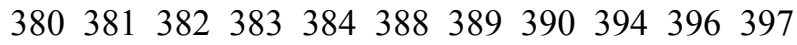

398399400401402408409410413418419

$\begin{array}{llllllll}423 & 424 & 425 & 426 & 435 & 452 & 460 & 461\end{array}$

$\begin{array}{lllllllllll}2 & 4 & 12 & 15 & 18 & 19 & 20 & 21 & 23 & 24 & 25\end{array}$

$\begin{array}{lllllllllll}26 & 27 & 29 & 31 & 36 & 37 & 38 & 41 & 42 & 46 & 47\end{array}$

2

50

$\begin{array}{lllllllllll}48 & 49 & 50 & 51 & 54 & 59 & 60 & 68 & 69 & 70 & 72\end{array}$

$\begin{array}{lllllllllll}74 & 76 & 77 & 78 & 81 & 86 & 89 & 90 & 91 & 92 & 93\end{array}$

$111 \quad 112113 \quad 187 \quad 193 \quad 195$

$458459465466468470472476477478 \quad 480$

481482483484486489490491492493494

495496497498499500501502503504505

3

75

506509510511512513514515517518519

$\begin{array}{lllllllllll}520 & 521 & 522 & 523 & 525 & 529 & 530 & 531 & 532 & 533 & 534\end{array}$

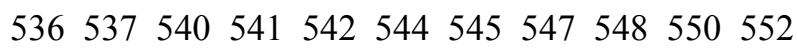

$\begin{array}{lllllllll}553 & 555 & 556 & 559 & 560 & 562 & 570 & 578 & 722\end{array}$

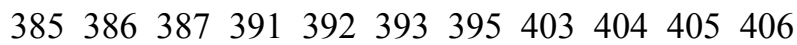

407411412414415416417420421422427

4

64 $\begin{array}{lllllllllll}428 & 429 & 430 & 431 & 432 & 433 & 434 & 436 & 437 & 438 & 439\end{array}$ $\begin{array}{lllllllllll}440 & 441 & 442 & 443 & 444 & 445 & 446 & 447 & 448 & 449 & 450\end{array}$ 451453454455456457462463464467469 
$\begin{array}{lllllllll}471 & 473 & 474 & 475 & 479 & 485 & 487 & 488 & 721\end{array}$

$\begin{array}{lllllllllll}13 & 39 & 43 & 44 & 57 & 61 & 67 & 79 & 123 & 127 & 128\end{array}$

$\begin{array}{lllllllllll}129 & 131 & 133 & 134 & 135 & 136 & 139 & 140 & 142 & 143 & 144\end{array}$

5

43

$\begin{array}{lllllllllll}145 & 147 & 148 & 149 & 150 & 152 & 157 & 159 & 160 & 163 & 165\end{array}$

$\begin{array}{llllllllll}166 & 170 & 171 & 172 & 174 & 175 & 176 & 180 & 190 & 214\end{array}$

$\begin{array}{lllllllllll}704 & 713 & 715 & 716 & 717 & 728 & 731 & 733 & 734 & 741 & 747\end{array}$

753760761764765766768771774775776

$\begin{array}{lllllllllll}777 & 778 & 779 & 781 & 782 & 785 & 787 & 788 & 791 & 792 & 793\end{array}$

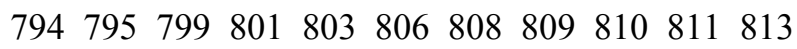

6

97

$\begin{array}{lllllllllll}831 & 833 & 837 & 839 & 842 & 843 & 844 & 846 & 847 & 849 & 850\end{array}$

$\begin{array}{lllllllllll}854 & 856 & 858 & 859 & 861 & 862 & 863 & 864 & 866 & 868 & 873\end{array}$

$\begin{array}{lllllllllll}874 & 876 & 878 & 879 & 883 & 884 & 885 & 886 & 887 & 888 & 891\end{array}$

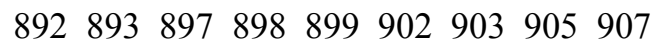

724725726727729730732735736737738

$\begin{array}{lllllllllll}739 & 740 & 742 & 743 & 744 & 745 & 746 & 748 & 749 & 750 & 751\end{array}$

$752754755756757758759762763767 \quad 769$

7

75

$\begin{array}{lllllllllll}770 & 772 & 773 & 780 & 783 & 784 & 786 & 789 & 790 & 796 & 797\end{array}$

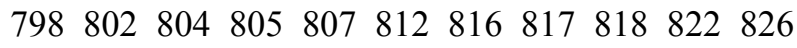

$\begin{array}{lllllllllll}832 & 834 & 835 & 836 & 838 & 840 & 841 & 851 & 853 & 860 & 865\end{array}$

$\begin{array}{lllllllll}869 & 870 & 871 & 872 & 877 & 880 & 881 & 882 & 894\end{array}$

8

51

$\begin{array}{lllllllllll}97 & 102 & 104 & 106 & 108 & 109 & 114 & 116 & 117 & 121 & 122\end{array}$ 
$\begin{array}{lllllllllll}124 & 125 & 126 & 130 & 132 & 137 & 141 & 153 & 154 & 158 & 161\end{array}$

$\begin{array}{lllllllllll}167 & 168 & 173 & 178 & 179 & 181 & 185 & 196 & 204 & 208 & 209\end{array}$

210218224244257265266269271278280 294301303304319325333

$\begin{array}{lllllllllll}118 & 183 & 184 & 188 & 189 & 191 & 192 & 197 & 198 & 199 & 200\end{array}$ 201203206207211212213215216217219

$\begin{array}{lllllllllll}220 & 221 & 223 & 227 & 229 & 230 & 231 & 232 & 233 & 235 & 236\end{array}$

9

58

241267270273283284291296300305310

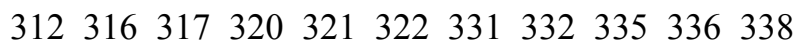

340343345

$\begin{array}{lllllllllll}663 & 664 & 671 & 679 & 680 & 681 & 683 & 684 & 685 & 686 & 687\end{array}$ $\begin{array}{lllllllllll}688 & 693 & 698 & 700 & 701 & 702 & 707 & 711 & 714 & 718 & 719\end{array}$

10

49

890895896900901906908909910911912

913914916918920

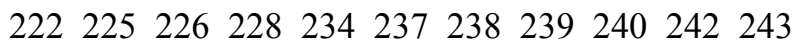

$\begin{array}{lllllllllll}246 & 247 & 248 & 249 & 250 & 252 & 253 & 254 & 255 & 258 & 259\end{array}$

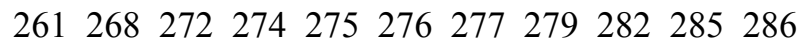

11

73

287288289290292293295297298299302

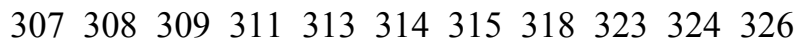

$\begin{array}{lllllllllll}327 & 328 & 329 & 330 & 337 & 339 & 341 & 344 & 346 & 347 & 349\end{array}$

351353354355357358361 
$\begin{array}{lllllllllll}1 & 7 & 10 & 11 & 14 & 16 & 17 & 22 & 28 & 30 & 32\end{array}$

12

22

$\begin{array}{lllllllllll}33 & 34 & 35 & 40 & 45 & 55 & 56 & 58 & 62 & 63 & 146\end{array}$

507508516524526527528535538539543

546549551554557558561563564565566

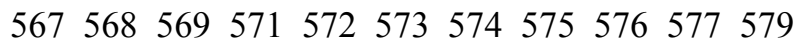

13

60

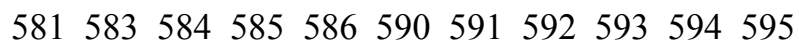

$\begin{array}{lllllllllll}596 & 597 & 598 & 599 & 600 & 602 & 609 & 613 & 623 & 625 & 635\end{array}$

$636 \quad 649650 \quad 653 \quad 723$

$\begin{array}{lllllllllll}3 & 5 & 6 & 8 & 9 & 52 & 53 & 64 & 65 & 66 & 71\end{array}$

$\begin{array}{lllllllllll}73 & 75 & 80 & 82 & 83 & 84 & 85 & 87 & 88 & 94 & 95\end{array}$

14

52

$\begin{array}{lllllllllll}96 & 98 & 99 & 100 & 101 & 103 & 105 & 107 & 110 & 115 & 119\end{array}$

$\begin{array}{lllllllllll}120 & 138 & 151 & 155 & 156 & 162 & 164 & 169 & 177 & 182 & 186\end{array}$

194202205281306915917919

$\begin{array}{lllllllllll}580 & 582 & 587 & 588 & 589 & 601 & 603 & 604 & 605 & 606 & 607\end{array}$ $\begin{array}{lllllllllll}608 & 610 & 611 & 612 & 614 & 615 & 616 & 617 & 618 & 619 & 620\end{array}$ $\begin{array}{lllllllllll}621 & 622 & 624 & 626 & 627 & 628 & 629 & 630 & 631 & 632 & 633\end{array}$ $\begin{array}{lllllllllll}634 & 637 & 638 & 639 & 640 & 641 & 642 & 643 & 644 & 645 & 646\end{array}$ 15 88 $647648 \quad 651652654 \quad 655 \quad 656 \quad 657 \quad 658 \quad 659660$ $661662665666 \quad 667668669670672673 \quad 674$ $\begin{array}{lllllllllll}675 & 676 & 677 & 678 & 682 & 689 & 690 & 691 & 692 & 694 & 695\end{array}$ 696697699703705706708709710712904 
Table S4 Sensor placement solution in various failure scenarios for the HaiN town.

\begin{tabular}{|c|c|c|c|c|c|c|c|c|c|c|}
\hline Scenario & $\mathrm{c} 1$ & $\mathrm{c} 2$ & $\mathrm{c} 3$ & $\mathrm{c} 4$ & $\mathrm{c} 5$ & $\mathrm{c} 6$ & $\mathrm{c} 7$ & $\mathrm{c} 8$ & c9 & $\mathrm{c} 10$ \\
\hline 0 & 352 & 31 & 481 & 471 & 190 & 733 & 752 & 179 & 231 & 800 \\
\hline 1 & 352 & 31 & 481 & 488 & 190 & 887 & 732 & 265 & 236 & 906 \\
\hline 2 & 264 & 187 & 498 & 488 & 172 & 907 & 740 & 124 & 233 & - \\
\hline 3 & 352 & 47 & 560 & - & - & 785 & 752 & 125 & 336 & - \\
\hline 4 & - & 86 & - & - & 190 & 864 & 738 & 208 & 336 & - \\
\hline 5 & - & 47 & - & - & - & 777 & 752 & 124 & 283 & - \\
\hline 6 & - & 86 & 483 & - & 190 & - & 836 & 161 & 291 & - \\
\hline 7 & - & 46 & - & - & - & 887 & - & 122 & - & - \\
\hline 8 & 352 & 38 & 559 & - & - & 864 & - & 124 & - & - \\
\hline 9 & 352 & - & 560 & - & - & 829 & - & 137 & - & 800 \\
\hline 10 & 352 & - & 562 & 471 & 123 & - & 786 & - & - & - \\
\hline 11 & 352 & - & - & - & 123 & - & 739 & - & - & - \\
\hline 12 & - & 23 & - & 721 & 13 & & - & - & - & - \\
\hline 13 & - & 31 & - & - & 13 & - & - & - & - & - \\
\hline 14 & - & - & - & - & - & - & - & 265 & - & - \\
\hline Scenario & $\mathrm{c} 11$ & $\mathrm{c} 12$ & $\mathrm{c} 13$ & $\mathrm{c} 14$ & $\mathrm{c} 15$ & & & & & \\
\hline 0 & 346 & 22 & 584 & 164 & 637 & & & & & \\
\hline 1 & 268 & 35 & 568 & - & 622 & & & & & \\
\hline 2 & 353 & - & 594 & 82 & 624 & & & & & \\
\hline 3 & 346 & 35 & 623 & 83 & 637 & & & & & \\
\hline
\end{tabular}




$\begin{array}{llllll}4 & 288 & 30 & 577 & 84 & 637 \\ 5 & 329 & 35 & 613 & 84 & 622 \\ 6 & 346 & - & 594 & - & 612 \\ 7 & 253 & 30 & 609 & 83 & 665 \\ 8 & 282 & - & 561 & - & - \\ 9 & 288 & - & - & - & - \\ 10 & - & - & - & - & - \\ 11 & 295 & - & - & - & - \\ 12 & - & - & - & - & - \\ 13 & - & - & - & - & - \\ 14 & - & - & - & - & -\end{array}$

\section{Figures}

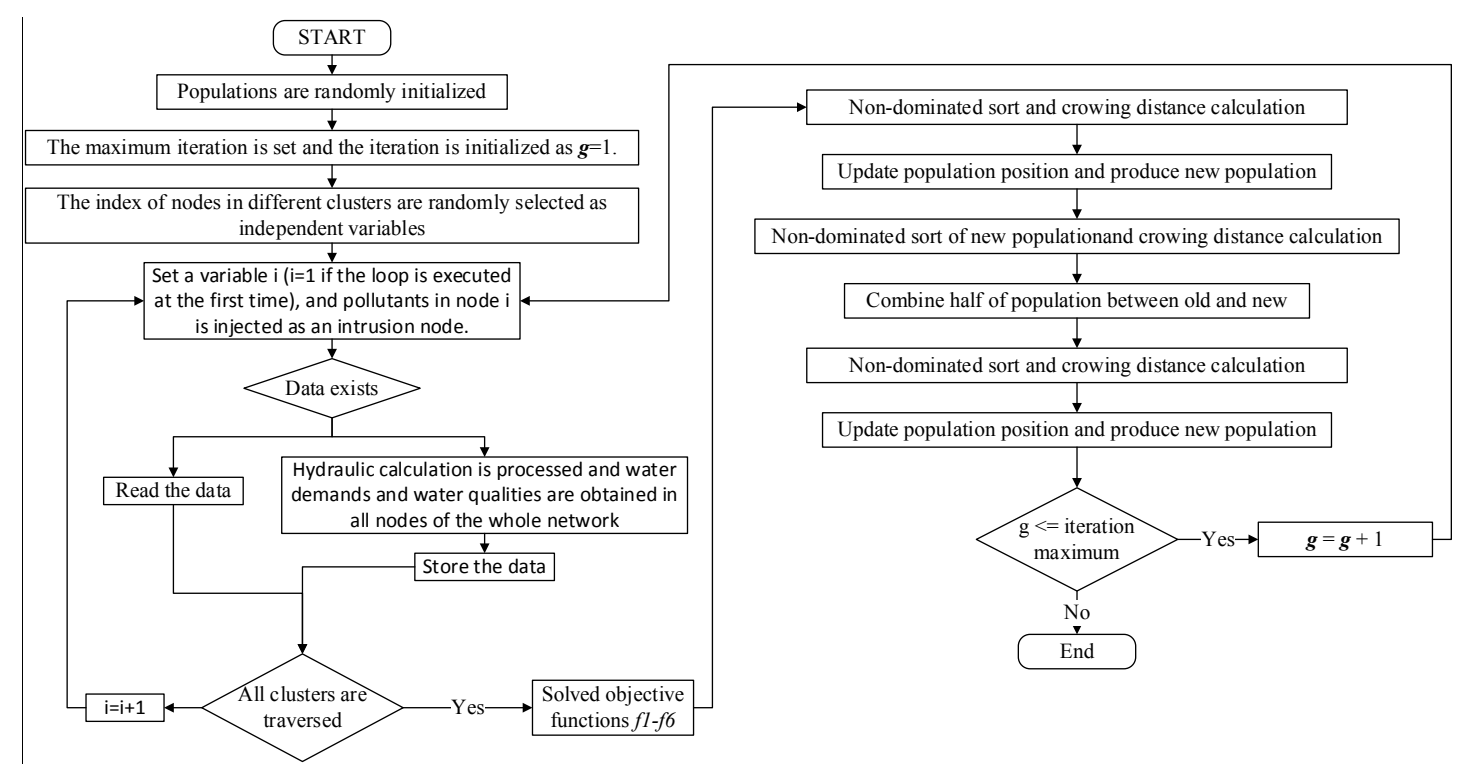

Figure S1 Flowchart of NSGA-II optimization. 


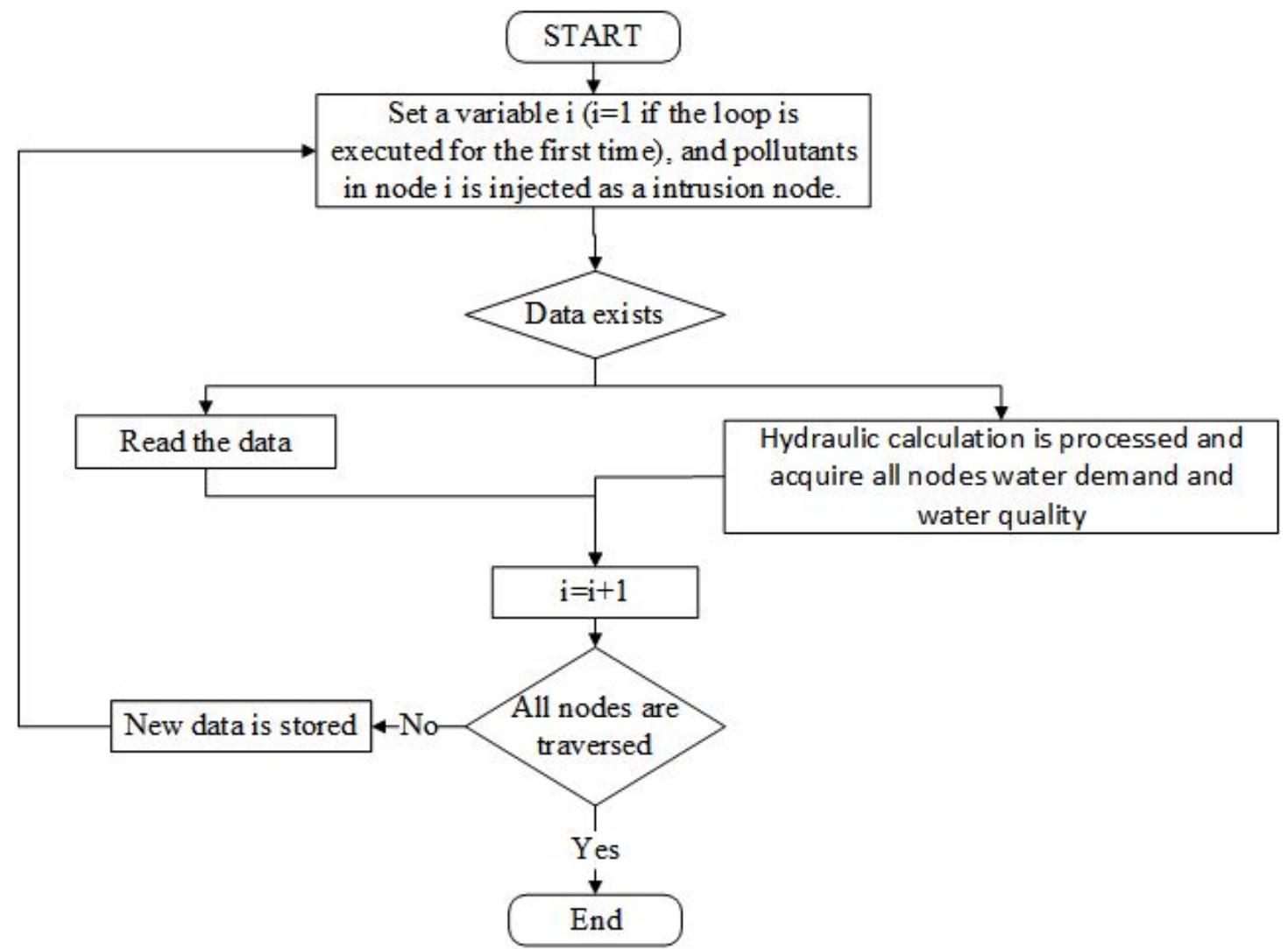

Figure S2 Flowchart of the backup archive method.

(a)

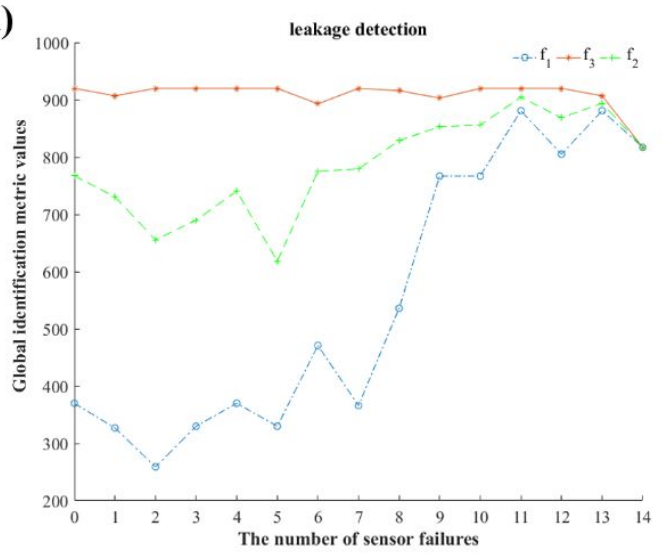

(b)

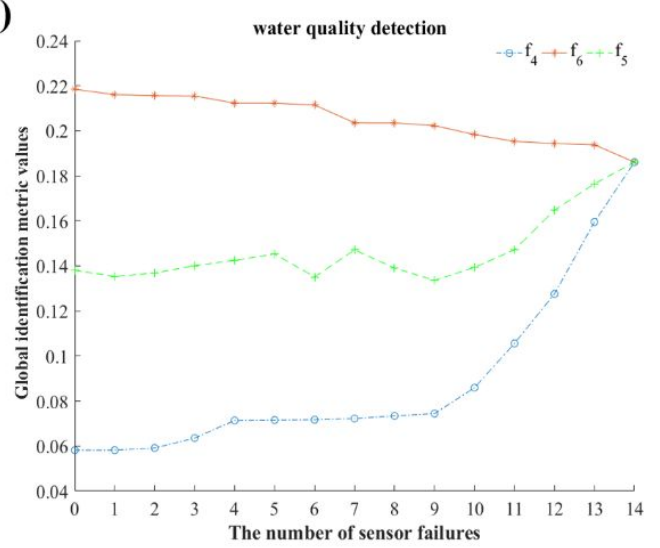

Figure S3 Global identification metric values for different sensor failure scenarios in HaiN town:

(a) leakage detection; (b) water contaminant intrusion detection. 


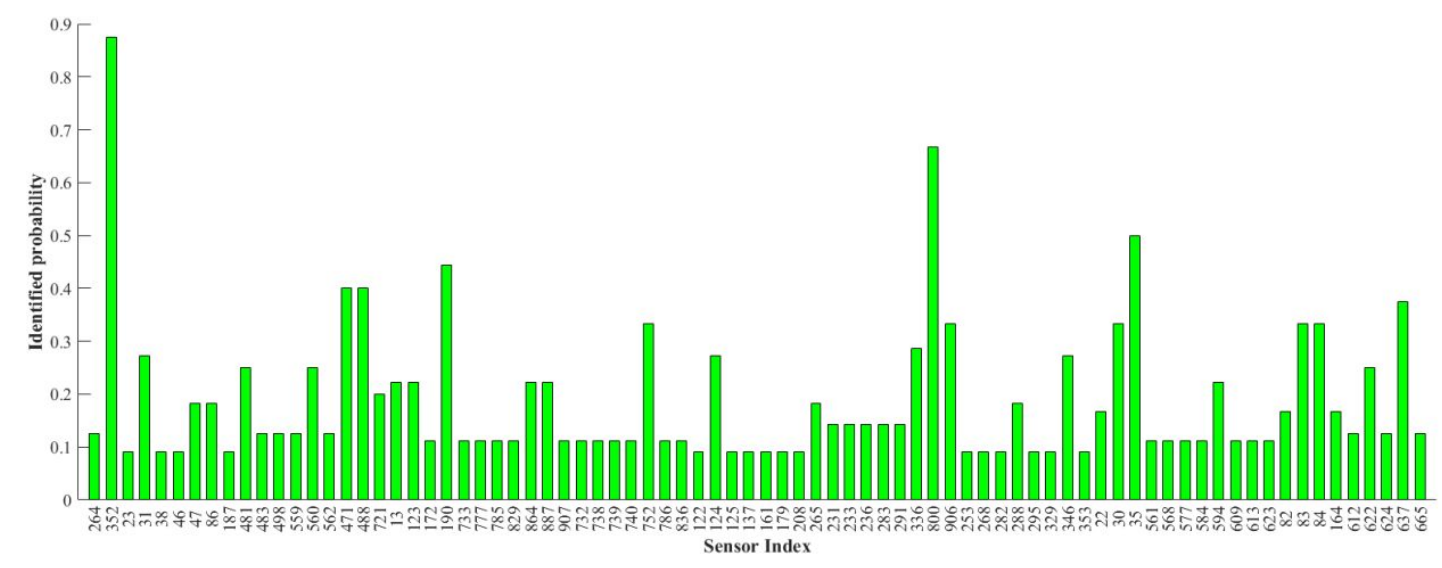

Figure S4 Sensor ranking in terms of the minimum global identification value across different failure levels for HaiN town.

(a)

(b)
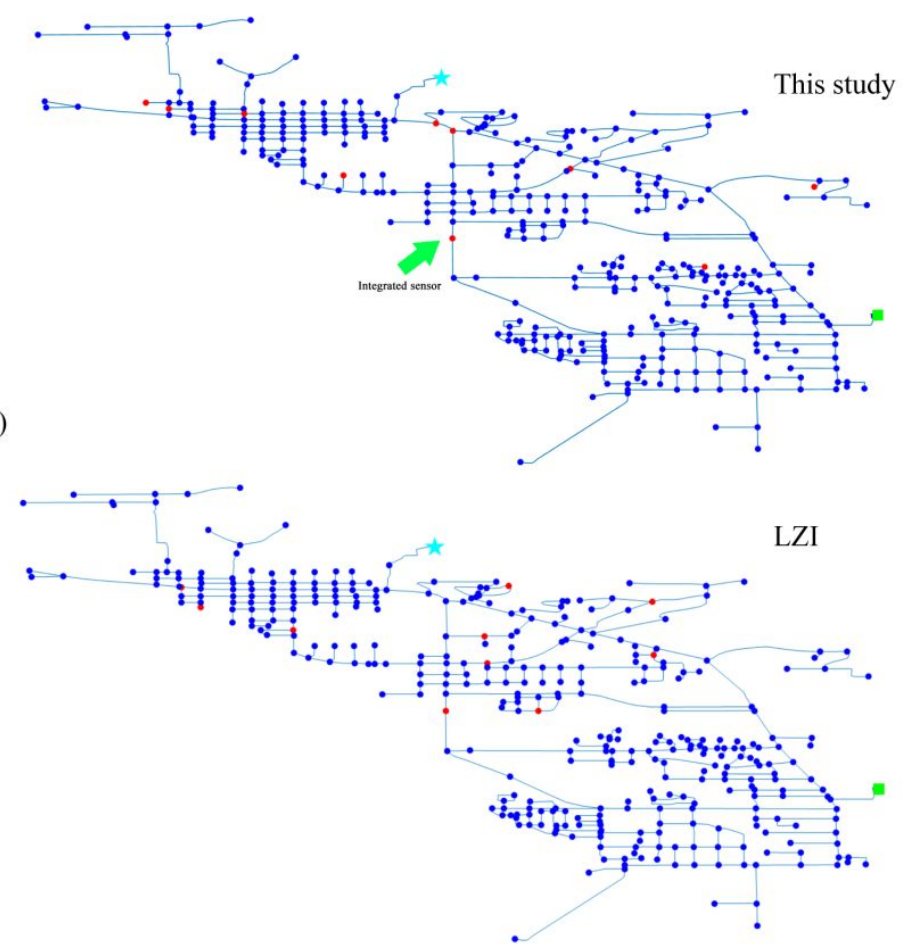

(c)

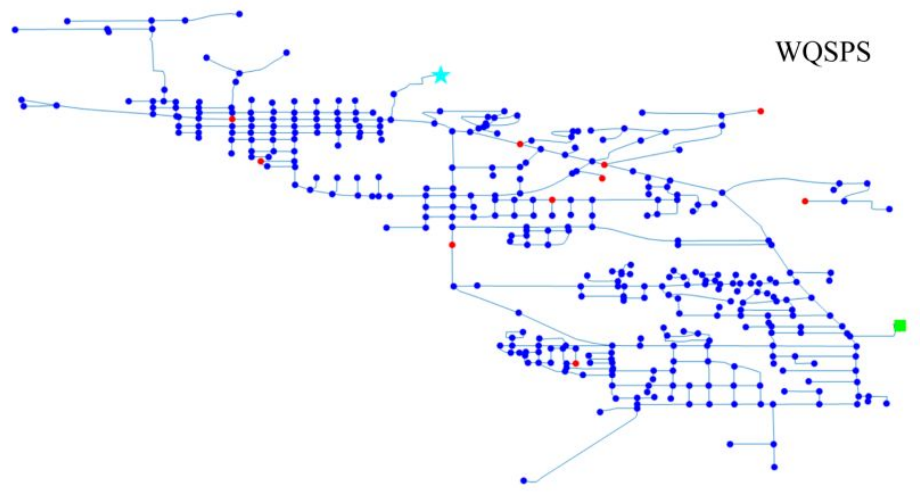


Figure S5 Locations of integrated sensors of EXA7 network: (a) this study; (b) LZI model; (c)

$$
\text { WQSPS model. }
$$

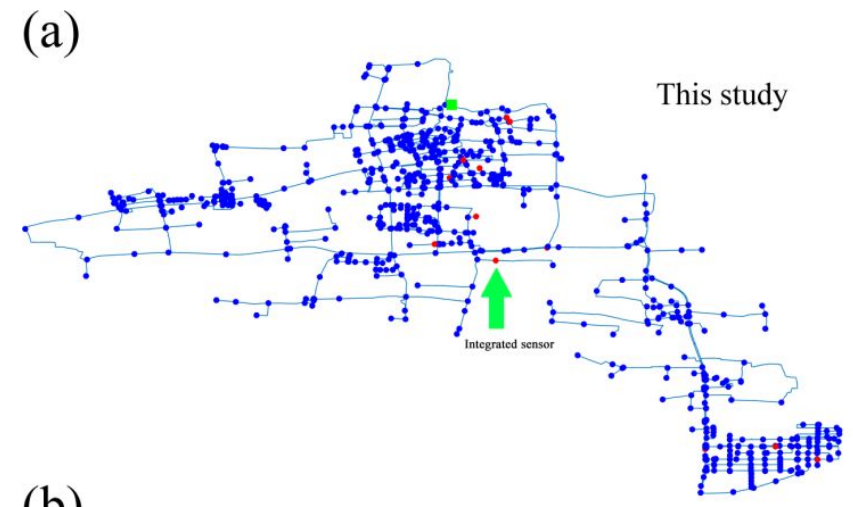

(b)

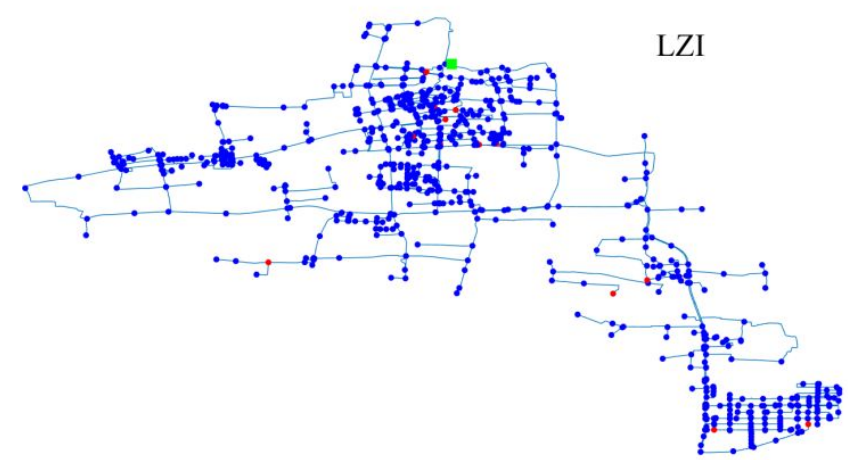

(c)

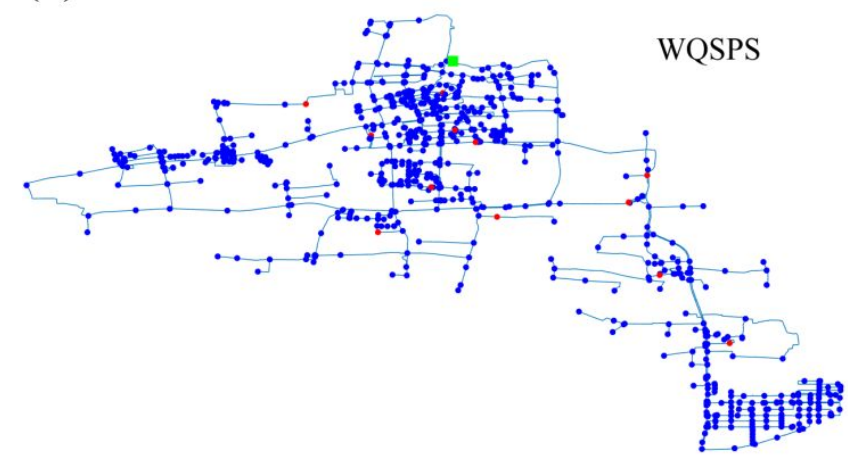

Figure S6 Locations of sensors of HaiN town network: (a) this study; (b) LZI model; (c) WQSPS model.

\section{S1. Trial-and-error principle}

This method aims to select the number of classes $c$ for clustering analysis. The trial-and-error 
principle means a simple back-and-forth test concerning the effect on different class numbers. In this study, the cost of leakage is applied to select the number of classes. The value of $c$ is equal to two at first. After the clustering is processed, junctions are divided into two classes. Then, PRVs are set to the boundary pipes connected with two junctions of different classes to isolate a class from others. In the WDS, the water flow condition in the PRV is turbulent. The head loss will be produced with a flow vortex. The head loss equation can be expressed as:

$$
h_{j}=\xi \frac{v^{2}}{2 g}
$$

Where $\zeta$ is the coefficient of local resistance; $v$ is flow velocity; $g$ is the gravitational acceleration.

The coefficient of local resistance is related to the local mutation form. Thus, the opening degree is related to the coefficient of local resistance and influence on the head loss of pipes. The coefficient of local resistance can be solved as an empirical formula of opening degree:

Table S5 Empirical formula between resistance coefficient and the opening degree of PRVs.

\begin{tabular}{cc}
\hline Diameters/mm & Functions $^{1}$ \\
\hline 100 & $\zeta=1.5875 \times 0.715^{1 / \beta} \times \beta^{-3.1677}$ \\
150 & $\zeta=1.2786 \times 0.937^{1 / \beta} \times \beta^{-2.284}$ \\
200 & $\zeta=0.8616 \times 1.1552^{1 / \beta} \times \beta^{-1.7721}$ \\
300 & $\zeta=0.906 \times 0.8477^{1 / \beta} \times \beta^{-2.449}$ \\
400 & $\zeta=0.7238 \times 0.8408^{1 / \beta} \times \beta^{-2.401}$ \\
\hline
\end{tabular}

The opening degree of parts of PRVs is set to $60 \%$ to reduce leakages due to each class's high 
internal pressure ${ }^{9}$. The resistance coefficient can be changed via adjusting the $\beta(0-100 \%)$. When the resistance is changed, the head loss of pipe will be changed following as Eq. S1. Then, the pressure head will be changed. The hydraulic condition is changed.

A leakage uniformity function is used as an objective function for optimizing the number of classes. The equation can be expressed as ${ }^{2}$ :

$$
\min F_{t}=f_{t} \sum_{k=1}^{N} L_{k} c_{k}\left[\frac{\left(H_{i}-z_{i}\right)+\left(H_{j}-z_{j}\right)}{2}\right]^{\alpha}
$$

Where $F_{t}$ is the total cost of loss of leakage; $f_{t}$ is the unit cost of loss of leakage that is set to $3.19 \mathrm{CNY} / \mathrm{m}^{3}$ in this study; $\alpha$ is the index of the leakage that is equal to $1.18^{2-5} ; c_{k}$ is the parameter of leakage that is equal to $2.5^{*} 10^{-6} ; N$ is the total of pipes; $H_{i}, z_{i}, H_{j}$ and $z_{j}$ are the total heads and ground elevation at junction $i$ and $j$.

Then, the value of $c$ is equal to 3. Junctions are divided into three classes. PRVs are set to the boundary pipes. The leakage uniformity function is solved, and the cost of loss of leakage is obtained. The value $c$ is equal to 4,5 , etc. The cost of loss of leakages is measured in the same way.

This principle is applied in Exa7 and HaiN town studies, shown in Figure 3-4. In Exa7, the range of $\mathrm{c}$ is from 2 to 25 . In HaiN town, the range of $\mathrm{c}$ is from 2 to 30 . The EPS period time is 24 hours. The results of Eq. S2 is shown in Figure S7. The minimum leakage cost is 196.92 CYN/day and 1569.33 CYN/day in Exa7 and HaiN town, respectively. The number of classes corresponding to 10 and 15 , respectively. 
(a)

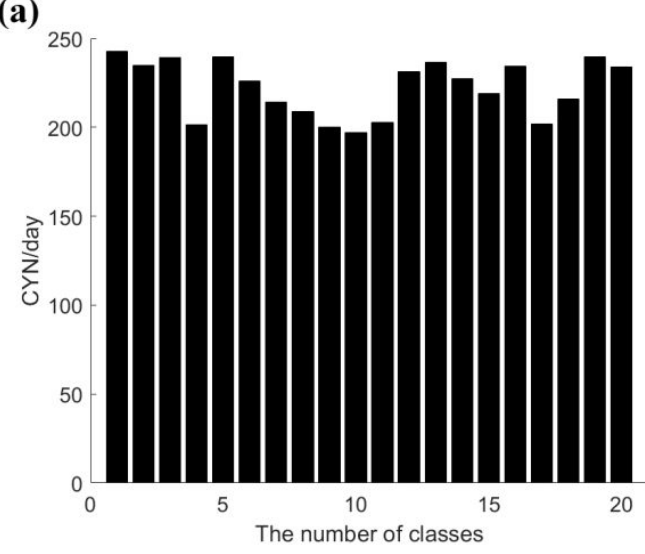

(b)

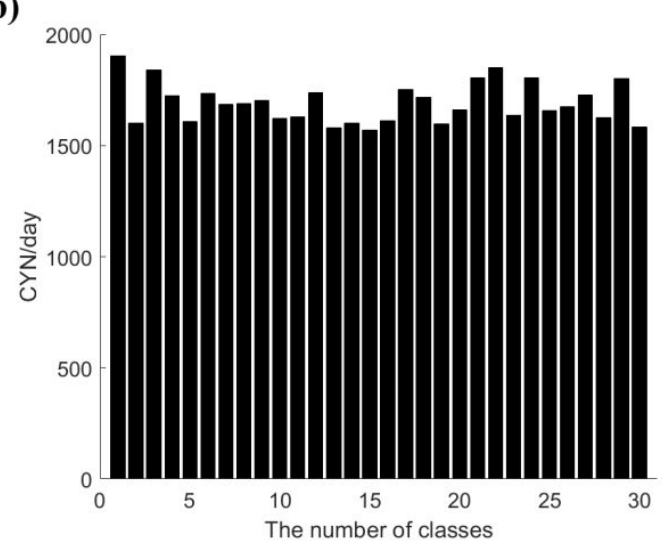

Figure S7 The relationship between the cost of leakages and classes: (a) Exa7; (b) HaiN town.

\section{S2. Comparison of the clustering model}

Three clustering models are employed to classify the junctions, including K-means, FCM, and Gaussian mixture model (GMM). The detecting objective is the number of outlier groups. When we have completed the clustering process, junctions are divided into different classes. A few junctions belonging to one class are not connected with other junctions. These junctions are called outliers, shown in Figure S8, which are eliminated as reference ${ }^{6}$. The model has a minimum number of outlier groups that can be regarded as effective clustering results, representing a close connection between compactness of junction locations and pressure change. The fewer number of outlier groups mean junctions in each class are tightly set in close location. In the following process, the integrated sensors will more uniformly be distributed in the WDS. This model is applied in Exa7 and HaiN town case studies, shown in Figure 3-4. The number of classes is 10 and 15, respectively. The results are shown in Table S6, where FCM has minimum outliers in both Exa7 and HaiN town cases. 


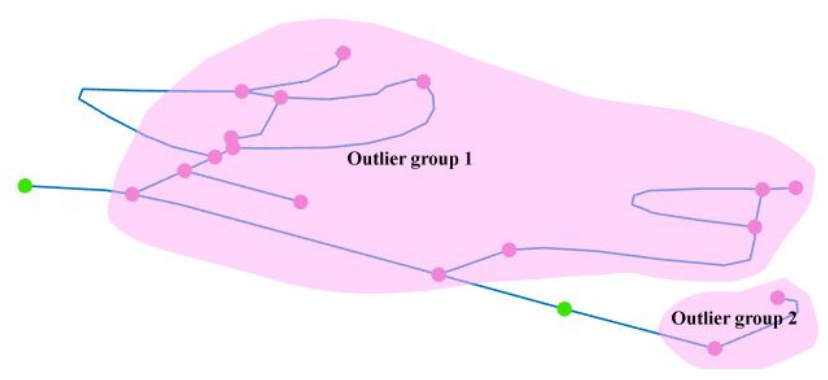

Figure S8 Outlier groups.

Table S6 The number of outliers in three models.

\begin{tabular}{ccc}
\hline Models & Exa7 & HaiN town \\
\hline K-means & 201 & 325 \\
FCM & 72 & 116 \\
GMM & 130 & 247 \\
\hline
\end{tabular}

Meanwhile, the runtime of the three models is solved, shown in Table S7. The number of partitions is 10 and 15, respectively, in Exa7 and HaiN town.

The model having the fastest runtime is K-means. However, the outliers in K-means are more than FCM and GMM. The fewest outliers appear in the FCM model, and it has a moderate runtime (less than GMM and larger than K-means) but not much difference among them that can be ignored. For the minimization of outliers, the FCM is select as the cluster model in this study.

Table S7 The run time of three models. 
Model The runtime of Exa7 (s) The runtime of HaiN town (s)

\begin{tabular}{lcc} 
K-means & 0.111 & 0.209 \\
FCM & 0.592 & 9.511 \\
& & \\
GMM & 1.724 & 13.415 \\
\hline
\end{tabular}

\section{S3. A case of the backup archive}

In this way, the calculated time will be reduced, and effectiveness is improved. For instance, the network of case two (in the case studies section) has 920 junctions. In each EA iteration, water quality and water demand at 22160 scenarios $(920 \times 23)$ should be calculated, and each junction's results should also be acquired. The total cycle in each iteration is $19467200(22160 \times 920)$, which has hugely wasted computation resources. Only 22160 times cycle can be executed in the backup archive if all results are stored in memory, cutting 920 times computation. Therefore, the backup archive can be used effectively in large-scale networks. In short, compared with the traditional method "data archives", This backup archive does not need to calculate solutions of water demand and water quality of all nodes. We calculate the nodes that appeared in the NSGA-II process only once.

A run time analysis is processed to compare the procedure of backup archive versus data archive, shown in Table S8. The program speed using backup archive has a considerable improvement.

Table S8 The run time analysis on the backup archive and data archive models. 


\begin{tabular}{ccc}
\hline Cases & Backup archive & Data archive \\
\hline Runtime of Exa7 $(d)$ & $3 d 14 h$ & $4 d 7 h$ \\
Runtime of Exa7 with & $5 d 21 h$ & $6 d 2 h$ \\
WQSPS & $4 d 5 h$ & $13 d 21 h$ \\
The runtime of HaiN town $(d)$ & $14 d 14 h$ & $17 d 21 h$ \\
The runtime of HaiN town & & \\
\hline with WQSPS $(d)$ & & \\
\hline
\end{tabular}

$d$ represents days.

$h$ represents hours.

\section{S4. Example of non-dominated sorting}

The non-dominated sorting (NS) method is a low-complexity procedure that requires two parameters as prerequisites: the domination count $n_{p}$ and the domination solutions $S_{p}$. Furthermore, the NS method with a crowded-comparison approach can maintain the diversity of samples to prevent the loss of results in terms of local optimized values. For instance:

Assume as 1000 number of population, which can be defined as:

$$
P=\left[\begin{array}{ccc}
f_{1}^{1} & \cdots & f_{6}^{1} \\
\vdots & \ddots & \vdots \\
f_{1}^{1000} & \cdots & f_{6}^{1000}
\end{array}\right]
$$

Where $f_{i}^{j}$ is the value of the objective function $f_{i}$ in the population of $j$, and the arranging 
strategy is $f_{1}, f_{5}, f_{3}, f_{4}, f_{2}$, and $f_{6}$.

The domination count $n_{p}$ is initialized as zero, and one of the best populations is looked up if objection function $f_{l}$ is better than others. If multiple best $f_{l}$ appear in matrix $P$, objection function $f_{2}$ is compared. If multiple best $f_{1}$ and $f_{2}$ exist, $f_{3}$ is compared, and so on. Then, set the best value to $P_{l}$, moved to the first row of matrix $P$. The rank of $P_{l}$ is set to 1 . Next, others are transferred to a collection $S_{l}$. Traversing each $S_{l}$ all of the individuals (the number of individuals is $n_{l}$ ) to search for the best solution in $S_{1}$, execute $n_{p}=n_{p}$ - 1 . If $n_{l}=0$, save it into set $Q$. Then, assigning $P_{2}=Q$ and putting others into a collection S2, except for $P_{1}$ and $P_{2}$. Then, search for the best solution in $S_{2}$, and then execute $n_{p}=n_{p}-1$. If $n_{l}=0$, save it into set $Q$. Repeat this process until $P_{n}$ is a null set.

For instance, assuming $P$ is a matrix $3 \times 3$ (three objection functions and three population): $P=\left[\begin{array}{lll}3 & 4 & 6 \\ 4 & 5 & 7 \\ 5 & 5 & 8\end{array}\right]$. Because all function values in row 1 are less than row 2 and row 3 , initialize $n_{p}$ to 0 . Row 2 and row 3 are transferred to the $S_{l}=\{[4,5,7],[5,5,8]\}$ and Row 1 is transferred to $F_{1}$ $=\{[3,4,6]\}$.

Then, element in $F_{l}$ is marked as rank 1 . In addition, row 2 is less than row 3 but all larger than row 1 , so $n_{2}$ is equal to 1 and $S_{2}=\{[5,5,8]\}$. Row 3 is larger than row 1 and row 2 , so $n_{3}$ is equal to $2 . F_{l}$ is not a null set, thus traverse $S_{1}$. Due to $[4,5,7]$ better than $[5,5,8]$. Thus, $Q=\{[4,5,7]\}$. Then, execute $n_{2}-1=1-1=0$. Mark element in matrix Q (row 2) to rank 2. Due to $n_{3}-1=2-1=$ $1>0, F_{2}=Q$ and traverse $S_{2}$. Execute $n_{3}-1=1-1=0$. So, $\mathrm{Q}=\{[5,5,8]\}$ and mark element of row 2 to rank 3. $F_{3}=Q$, and traverse $S_{3}$. Because $S_{3}$ is a null set, so Q is become a null set. $F_{4}=Q$ is null. At last, row 1, row 2 and row 3 are ranked as 1, 2, and 3.

After all the population calculations finish, NS is processed ${ }^{7}$ for sorting by the quality of results. 
Then, the crowding distance should be assigned to the whole matrix. For each population, the matrix rows have been sorted in rank. Then, all the results are sent into a collection for the sorting process at the following EA iteration process. Calculation of the crowding distance for the population is permission. The crowding distance equation is as Eq. $\mathbf{S 4}$.

$$
n_{d}(i)=n_{d}(i)+\left[f_{m}(i+1)-f_{m}(i-1)\right](\mathrm{S} 4)
$$

Where $n_{d}$ is the crowding distance and $f_{m}$ is the objective function $m$.

Next, the new rank and crowding distance should be calculated for each population position to be updated, using copy, cross, and mutation methods like the simple GA. Half of the old population and half of the new population are combined, which are all half of higher elements.

For instance: assuming the old population is: $P=\left[\begin{array}{lll}3 & 4 & 6 \\ 4 & 5 & 7 \\ 5 & 5 & 8\end{array}\right]$. Meanwhile, assuming the new population is: $P=\left[\begin{array}{lll}1 & 2 & 3 \\ 3 & 4 & 5 \\ 3 & 6 & 6\end{array}\right]$, the first row is rank one, the second is rank two, and the third is rank 3. The first row of old population and the first and second row of new populations are combined, while others are excluded. A new combination can be produced: $P=\left[\begin{array}{lll}1 & 2 & 3 \\ 3 & 4 & 5 \\ 3 & 4 & 6\end{array}\right]$. Finally, NS will be continued processing with the new population until reaching the maximum iteration times.

\section{S5. K-means model}

The K-means clustering algorithm is similar to the FCM. For partitioning $M$ data into $c$ clusters, Three steps should be implemented.

(1) The normal pressures of all junctions are solved with the hydraulic calculation process. 
(2) Pressure sensitivity analysis is conducted based on changes in the water demand. A leakage location is added at a junction, where an increase in water demand is induced. Then, the pressures at all junctions change correspondingly. The pressure difference between the leakage and normal states can be simply expressed as Eq.1. Moreover, the standardization and normalization are processed by Eq.2-3. Finally, a sensitivity analysis matrix for the pressure differences at all junctions can be obtained, shown as Eq.4.

(3) Use the sensitivity analysis matrix of the columns and divide the data into $c$ partitions. In this process, the top ten columns are assumed as the center of partitions $\boldsymbol{v}_{j}$. Eq. $\mathbf{S 5}$ calculates the distances between center points and others.

$$
d_{i j}=\frac{1}{M} \sum_{i=1}^{M}\left(\Delta \boldsymbol{p}_{i j}^{\prime \prime}-\boldsymbol{v}_{i j}\right)^{2}(\mathrm{~S} 5)
$$

Then, assign each column to the nearest partition with respect to the distance $\boldsymbol{d}$. Then, update the center $\boldsymbol{v}_{j}$ with the average of all columns in each partition. Repeat the step and update the center until the center does not change or up to the convergence condition.

\section{S6. GMM model}

Gaussian Mixture Model is a clustering model using Gaussian probability density function. GMM is fitted by classifying data points to the multivariate normal components that maximize the posterior component probability given the data. The GMM can be defined as Eq.S6.

$$
p(x)=\sum_{k=1}^{K} \pi_{k} p(x \mid k)
$$

Where $K$ is the number of mixture model; $\pi_{k}$ is the weight of $k$ th mixture model; $p(x \mid k)$ is the probability density function of the $k$ th model that the sample mean, and variance is $\mu_{k}$ and $\sigma_{k}$.

The sum of the $p(x)$ represents the probability that the sample belongs to each class. The 
equation can be expressed as Eq.S7.

$$
\max \sum_{k=1}^{c} p(x)
$$

In general, there is a sum in the logarithm function. It is challenging to solve the equation set by taking the derivative. So, a solution method is the EM algorithm is employed, which is divided into two steps. The first step is to generate the weight of each Gaussian model, assuming that the parameters of each Gaussian model are known. It can be initialized randomly or based on the results of the previous iteration. The second step is to go back to determine the parameters of the mixture model based on these weights. Repeat these two steps until the fluctuation is slight and approximate to the extreme value. The details of this algorithm refer as ${ }^{8}$.

\section{S7. Details of comparison}

\section{S7.1. Reasons for comparison}

The principal reason for this is that the proposed model is an enhanced version of the two models. Its leakage detection is an improvement on LZI, and its water quality detection is an improvement on WQSPS. Moreover, the LZI and WQSPS models have also been modified to meet the requirements of integrated sensors.

In the LZI model, a sensitivity matrix is generated in the same way as that shown in Eq. 5. Further, junctions are divided into different classes by the K-means model. The EA module is applied to optimize the sensor placements with three objective functions, $f_{1}-f_{3}$. After that, the $f_{4}$ $f_{6}$ are solved. In the case study on EXA7, ten classes are generated, and ten sensors are selected in each class. In HaiN town, 15 sensors are selected. 
The WQSPS model is a similar technique to that used in this study; however, clustering analysis is omitted. The sensors are selected randomly in all junctions. Then, the EA module is employed to solve the objective functions $f_{1^{-}} f_{6}$, based on a previous study ${ }^{10}$. The sensor failure scenarios are then created and optimized. In addition, the data archiving method is employed to speed up the EA optimization. This method builds up a database of intrusion time, detection time, and global intrusion identification metrics before optimization. In the case study of EXA7, ten sensors are randomly selected in 381 junctions. In HaiN town, 15 sensors are set in 920 junctions.

\section{S7.2. HaiN town results}

The indexes of sensors are $[371,27,560,463,139,788,804,130,198,896,252,33,583,87$, 661] for the LZI and $[352,26,493,456,145,824,812,137,345,701,228,146,613,95,624]$ for the WQSPS model. The locations of sensors for the LZI and WQSPS models are shown in Figure S6 (b)-(c). The $f_{6}$ in this study has the highest value, which suggests that it detects water contaminant intrusion the best. $f_{4}$ and $f_{5}$ in this study are the largest values, indicating that the stability of water contaminant intrusion detection is good. $f_{3}$ in this study is slightly lower than that of the WQSPS model but higher than that of the LZI. The $f_{l}$ is larger than that for LZI and WQSPS, so the probability of leakage detection is lower than that of LZI and WQSPS, but can reach the same effect if no sensors fail. The HaiN town case study involves a large-scale network, where sensors will be better protected. Therefore, the failure scenarios could be so few that $f_{l}$ has only a slight influence on network stability. The LZI model has a relatively poor effect on leakage detection for large-scale networks owing to its low $f_{2}$ and $f_{3}$. The stability of water quality detection is the best for the model in this study in terms of $f_{6}$. Furthermore, in addition to $f_{1}$, all its objective functions are superior to 
those of other models.

\section{S8. The original data}

The original data of flow rates and pattern multipliers in two cases are shown in the data sheet file (an excel file).

\section{S9. The system environment}

The specifications of the computer parameters are as follows: the CPU is i7-9700K; the RAM is DDR4-96G; the ROM is intel-SSD-1T; the operating system is Windows 10, and the MATLAB$2018 \mathrm{~b}$ software is used. 


\section{References}

(1) Wu Yuebin; Qu Shilin; Zhang Weijia; Zhao Hongbin. Local Head Loss Caused by Valves in Water Distribution Network. J. Harbin Inst. Technol. 2003, 35 (11), 1311-1313.

(2) GERMANOPOULOS, G.; JOWITT, P. LEAKAGE REDUCTION BY EXCESS PRESSURE MINIMIZATION IN A WATER SUPPLY NETWORK. Proc. Inst. Civ. Eng. 1989, 87 (2), 195-214. https://doi.org/10.1680/iicep.1989.2003.

(3) Giustolisi, O.; Savic, D.; Kapelan, Z. Pressure-Driven Demand and Leakage Simulation for Water Distribution Networks. J. Hydraul. Eng. 2008, 134 (5), 626-635. https://doi.org/10.1061/(ASCE)0733-9429(2008)134:5(626).

(4) Liberatore, S.; Sechi, G. M. Location and Calibration of Valves in Water Distribution Networks Using a Scatter-Search Meta-Heuristic Approach. Water Resour. Manag. 2009, 23 (8), 1479-1495. https://doi.org/10.1007/s11269-008-9337-6.

(5) Covelli, C.; Cozzolino, L.; Cimorelli, L.; Della Morte, R.; Pianese, D. Optimal Location and Setting of PRVs in WDS for Leakage Minimization. Water Resour. Manag. 2016, 30 (5), 1803-1817. https://doi.org/10.1007/s11269-016-1252-7.

(6) Mu, T.; Ye, Y.; Tan, H.; Zheng, C. Multistage Iterative Fully Automatic Partitioning in Water Distribution Systems. Water Supply 2020. https://doi.org/10.2166/ws.2020.288.

(7) Srinivas, N.; Deb, K. Muiltiobjective Optimization Using Nondominated Sorting in Genetic Algorithms. Evol. Comput. 1994, 2 (3), 221-248. https://doi.org/10.1162/evco.1994.2.3.221.

(8) Reynolds, D. A.; Quatieri, T. F.; Dunn, R. B. Speaker Verification Using Adapted Gaussian Mixture Models. Digit. Signal Process. 2000, 10 (1-3), 19-41. https://doi.org/10.1006/dspr.1999.0361. 
(9) Jiang B,; Zheng J.; Mu, T.; et al." Real-time pumps and valves integrated scheduling in water distribution system" Water \& Wastewater Engineering. 2018,54(11):103-107. https://10.13789/j.cnki.wwe1964.2018.0456. (in Chinese).

(10) Zhang, Q.; Zheng, F.; Kapelan, Z.; Savic, D.; He, G.; Ma, Y. Assessing the Global Resilience of Water Quality Sensor Placement Strategies within Water Distribution Systems. Water Res. 2020, 172, 115527. https://doi.org/10.1016/j.watres.2020.115527. 